\title{
Numerical Simulation and Theoretical Modeling of Transverse Compressive Failure in Fiber Reinforced Composite Materials
}

\author{
Takeaki Nadabe \\ Department of Advanced Energy \\ The University of Tokyo \\ Chiba, Japan \\ E-mail: nadabe@smart.k.u-tokyo.ac.jp
}

\begin{abstract}
This study investigates numerical simulation and theoretical modeling of transverse compressive failure in fiber reinforced composite materials. Firstly, numerical simulation of transverse compressive failure is conducted in order to understand the physical mechanism of the transverse compressive failure. The simulated results show that multiple shear bands appear in the material, and one of the shear bands develop to the entire material, and the transverse compressive failure is formed in the material. The material strength is associated with the initiation of shear band in the material due to localized stress concentration. Based on the mechanism in the numerical simulation, the theoretical modeling of transverse compressive failure is implemented. The model shows that the critical stress value of the material is represented by fiber volume fraction, matrix Poisson's ratio, matrix yield stress and angle of shear band.
\end{abstract}

Keywords-Numerical Simulation; Theoretical Modeling; Fracture Mechanism; Strength Analysis; Composite Materials

\section{INTRODUCTION}

Composite materials commonly have complex internal structures, including fibers, matrix, interfaces and interlaminar regions, and when precise evaluation of fracture strength of the material is conducted, the internal fracture process in the materials is necessary to be taken into account in the numerical analysis. In recent years, composite materials are being increasingly used in several industrial fields, and the precise evaluation of mechanical response of the material under various loading condition and environmental condition increases the necessity in design and improvement of industrial products [1-4]. Transverse compressive failure has been investigated in thirty decades for the experimental, theoretical and numerical approaches. Aragonés [5] conducted experimental investigation for transverse compressive failure particularly using scanning electron microscopy, and they stated that there are two important failure modes; namely matrix shear yielding and interfacial failure. Puck et al. [6] suggested the failure model for initiation of transverse compressive failure, which is based on MohrCoulumb fracture criterion, and they showed that the predicted fracture initiation under several combined transverse compression and shear stress states is very close to the experimental results, and the angle of fracture surface in fracture initiation is also obtained in the analysis.
Totry et al. [7] conducted numerical simulation for transverse compressive failure in both matrix shear yielding failure mode and interfacial failure mode, and they showed that the fracture initiation under several combined transverse compression and shear stress states is also predictable using the numerical simulation. In this study, the theoretical modeling of transverse compressive failure is investigated. The purpose of this study is to establish the numerical analysis method to analyze the transverse compressive strength of fiber reinforced composite materials. Firstly the numerical simulation of transverse compressive failure is conducted in order to understand how the material failure occurs. Then besed on the results of the simulation, the theoretical model is considered.

\section{NUMERICAL SiMULATION OF TRANSVERSE COMPRESSIVE FAILURE}

\section{A. Numerical Model}

Firstly the numerical simulation of transverse compressive failure is conducted. Figure 1 shows the numerical model of the transverse compressive failure. The white and gray elements in Figure 1 represent fibers and matrix, respectively. Here the fibers are modeled as the cross-section having the circle shape, and the matrix surrounds fibers. The length in both $\mathrm{x}$ and $\mathrm{y}$-direction is 60 $\mu \mathrm{m}$, and the thickness in $\mathrm{z}$-direction is $100 \mathrm{~mm}$. Each fiber and matrix is modeled by two-dimensional plate elements. The elements have eight nodes and four integration points in order to avoid the shear locking and zero-energy mode deformation particularly in plastic deformation. Fibers are placed randomly whose place are determined by generating random numbers in numerical calculation.

Due to the atomic structure in the inside of the fibers, the fibers commonly have the different material property in between fiber axial and transverse directions. In this analysis, the fibers are modeled as the isotropic elastic material in the inside of transverse plane, and transverse material property of the fiber is applied for the analysis. Table I shows the material property of the fibers. Carbon fiber AS4 (Hexcel Corp.) is assumed [8]. Matrix is modeled by isotropic elastic-plastic material. Commonly 


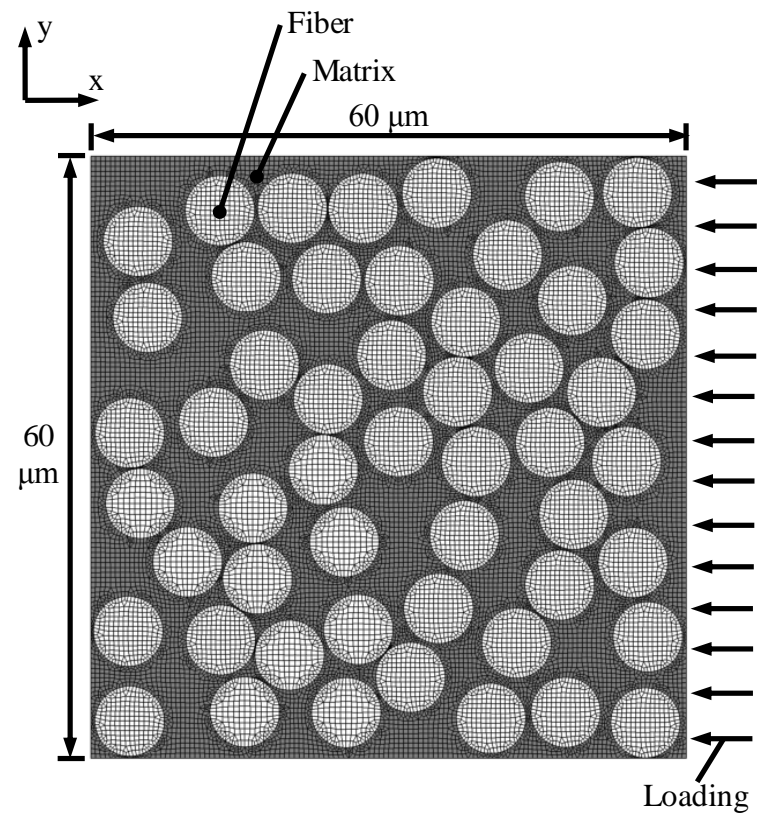

Figure 1. Numerical model of transverse compressive failure.

TABLE I. MATERIAL Property OF Fiber. CARBON FibER AS4 (HEXCEL CORP.) IS ASSUMED [8].

\begin{tabular}{|l|c|c|}
\hline Elastic modulus in transverse direction & 15 & $\mathrm{GPa}$ \\
\hline Poisson's ratio in transverse direction & 0.071 & \\
\hline Transverse shear modulus & 7.0 & $\mathrm{GPa}$ \\
\hline
\end{tabular}

TABLE II. MATERIAL PROPERTY OF MATRIX. EPOXY RESIN 3501-6 (HERCUles CHEMICAL COMPANY, INC.) Is ASSUMED [8].

\begin{tabular}{|l|c|c|}
\hline Elastic modulus & 4.2 & $\mathrm{GPa}$ \\
\hline Poisson's ratio & 0.34 & \\
\hline Yield stress & 90 & $\mathrm{MPa}$ \\
\hline
\end{tabular}

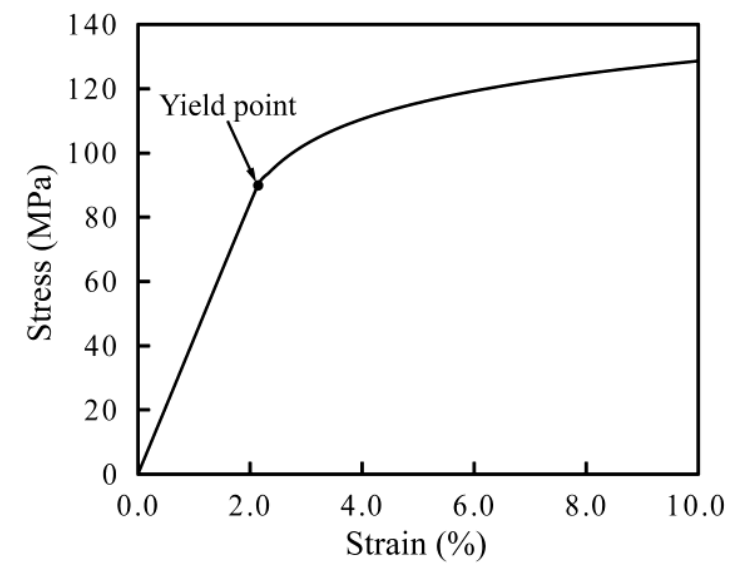

Figure 2. Stress-strain curve of matrix.

The transverse compressive failure of composite materials is affected by the nonlinear stress-strain relation of matrix, and in this analysis the nonlinear stress-strain curve of matrix shown in Figure 2 is applied, and the nonlinear finite element analysis is conducted. Table II shows the material property of matrix. Epoxy resin 3501-6 (Hercules Chemical Company, Inc.) Is assumed [8]. The quasi-static and room temperature environment are assumed in the

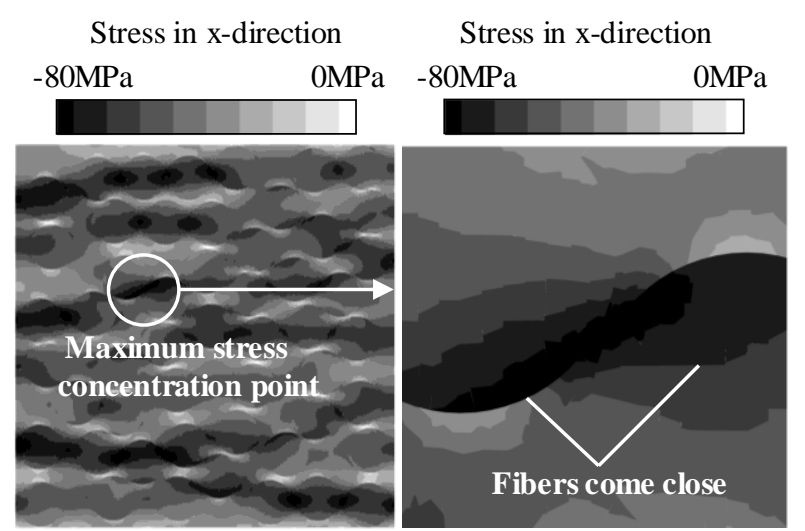

Average applied strain $0.60 \%$

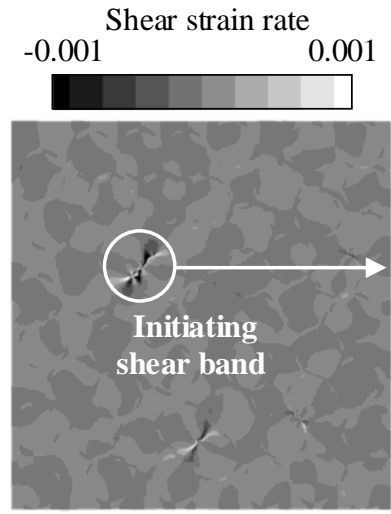

Average strain $1.14 \%$

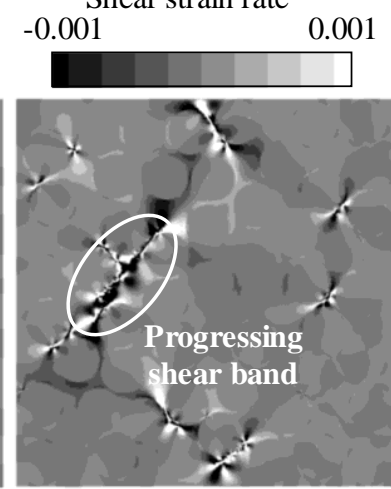

Average strain $1.15 \%$
Figure 3. Simulated results at initial state of loading.

Analysis. The geometrical nonlinear effect is also incorporated in the analysis. The incremental analysis in the finite element analysis is conducted by the arc-length method. In the initial increment, the average applied strain to the material in $\mathrm{x}$-direction is set to $0.004 \%$. The analysis is conducted until the average applied strain $10.0 \%$. The domain decomposition method is applied to conduct the parallel computing in the numerical calculation. The fortran program is produced for this analysis, and the analysis is conducted using this program.

\section{B. Simulated Results and Discussions}

Figure 3 shows the simulated results of the distribution of stress in $\mathrm{x}$-direction and shear strain rate at the initial state of loading. Because of the random placement of fibers, the stress concentration occurs in the material, and around the area where fibers come close, the high stress distributes. When the applied strain is increased, the stress in matrix around the maximum stress concentration point reaches the yield stress of matrix, and at this moment the matrix in this region starts to yield, and the deformation of matrix locally increases. After this moment, the shear stiffness of matrix reduces due to the yielding, and shear strain starts to increase and then the shear deformation of matrix in the local region increases. Due to this increase of shear deformation of matrix, the shear band initiates in the material at strain $1.14 \%$ from the maximum stress concentration point as shown in Figure 3, which is the rapid increase of shear deformation within a narrow band. As indicated in Figure 3, the shear band progresses through the 


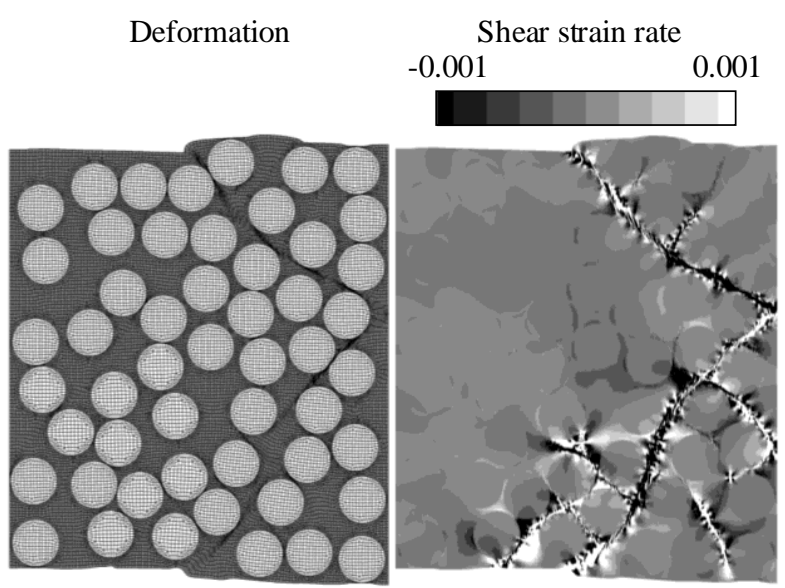

Average applied strain $6.0 \%$

Deformation Shear strain rate 0.001
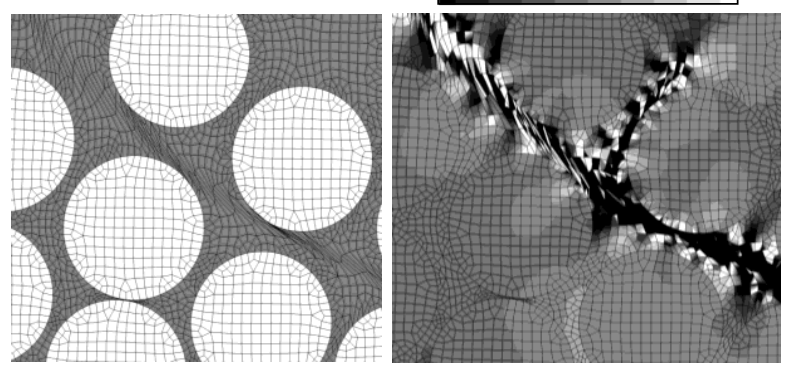

Average applied strain 6.0\% (Magnified picture)

Figure 4. Simulated results of transverse compressive failure.

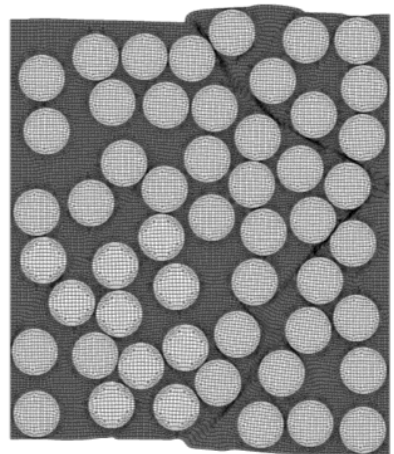

Simulated

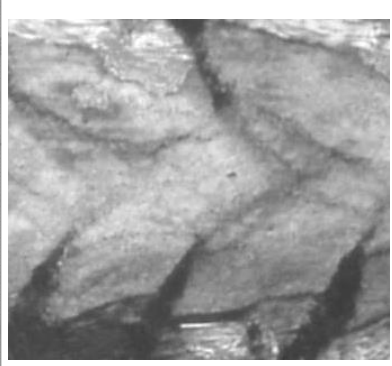

Experimental

(Microscope picture)
Figure 5. Comparison with experimental result.

fibers at strain $1.15 \%$.

Figure 4 shows the deformation and distribution of shear strain rate in the material after the damage development. In this state the failure is formed in the entire material. The place of shear band is seen to be different in between at strain $6.0 \%$ in Figure 4 and at strain $1.15 \%$ in Figure 3. At strain $1.15 \%$, the shear band is developed around the maximum stress concentration point, but because of the placement of fibers, this shear band is not developed to the entire material, as indicated in Figure 4. The fibers play the role of a wall to suppress the development of the shear band. On the other hand, the shear band of different place develops to the entire material, which behind initiates in the material. Considering the place of fibers, this shear band is easy to develop to the entire material. It is indicated that the first shear band is closely related with the maximum stress concentration, and the shear band developed to the entire material is closely related with the placement of fibers. As shown in Figure 5, the finally formed transverse compressive failure is close to the microscope picture in the experimental result.

\section{THEORETICAL MODELING OF TRANSVERSE COMPRESSIVE FAILURE}

In the previous investigation of this study, the theoretical models for the deformation behavior of fiber reinforced composite materials were obtained for the purpose of the analysis in the case of longitudinal compressive failure. The results are described in Ref. [9]. The fundamental mathematical equations are also applicable in the case of transverse compressive failure, and here firstly the fundamental mathematical equations are summarized. In Ref. [9], the fundamental equations expressing deformation of composite materials consist of motion equation and constitutive equation. The motion equation is represented as the following,

$$
\rho_{0} \frac{\partial^{2} u_{i}}{\partial t^{2}}=\frac{\partial P_{i j}}{\partial X_{j}}+\rho_{0} f_{i}
$$

where $\rho_{0}$ is density, ${ }^{t}$ is time, ${ }^{u_{i}}$ is displacement, $X_{j}$ is coordinate at reference configuration, $P_{i j}$ is the first Piola-Kirchhoff stress and $f_{i}$ is external force. The nonlinear stress-strain relation of composite materials is represented by the nonlinear deformation theory shown by Tohgo et al. [10].

$$
\begin{aligned}
& d \boldsymbol{\sigma}=\boldsymbol{C}_{\text {comp }} d \boldsymbol{\varepsilon} \\
& \boldsymbol{C}_{\text {comp }}=\boldsymbol{C}_{m}\left\{\left(1-V_{f}\right)\left(\boldsymbol{C}_{f}-\boldsymbol{C}_{m}\right) \boldsymbol{S}+\boldsymbol{C}_{m}\right\}^{-1} \boldsymbol{K} \\
& \boldsymbol{K}=\left(1-V_{f}\right)\left\{\left(\boldsymbol{C}_{f}-\boldsymbol{C}_{m}\right) \boldsymbol{S}+\boldsymbol{C}_{m}\right\}+V_{f} \boldsymbol{C}_{f}
\end{aligned}
$$

where $d \boldsymbol{\sigma}$ is stress rate, $d \boldsymbol{\varepsilon}$ is strain rate, $\boldsymbol{C}_{\text {comp }}, \boldsymbol{C}_{f}$ and $C_{m}$ are constitutive tensors of composites, fibers and matrix, respectively, $V_{f}$ is fiber volume fraction and $S$ is Eshelby tensor. For evaluating equivalent stress of matrix, the following relation is applied.

$$
d \boldsymbol{\sigma}_{m}=\boldsymbol{C}_{m}(\boldsymbol{S}-\boldsymbol{I}) \boldsymbol{K}^{-\boldsymbol{I}}\left\{\boldsymbol{C}_{m}+\left(\boldsymbol{C}_{f}-\boldsymbol{C}_{m}\right) \boldsymbol{S}\right\}(\boldsymbol{S}-\boldsymbol{I})^{-1} \boldsymbol{C}_{m}^{-1} d \boldsymbol{\sigma}
$$

where $d \boldsymbol{\sigma}_{m}$ is stress rate of matrix and $\boldsymbol{I}$ is unit tensor. Then the deformation of composite materials is expressed by the following two equations [9].

$$
\begin{aligned}
& \rho_{0} \frac{\partial^{2} u_{i}}{\partial t^{2}}=\frac{\partial P_{i j}}{\partial X_{j}}+\rho_{0} f_{i} \\
& \dot{P}_{i j}=J \frac{\partial X_{j}}{\partial x_{m}}\left(C_{i m k l}^{m a t}+\sigma_{l m} \delta_{i k}\right) \frac{\partial \dot{u}_{k}}{\partial x_{l}}
\end{aligned}
$$

where $C_{i m k l}^{m a t}$ is constitutive tensor in material description, $\sigma_{l m}$ is Cauchy stress, $\delta_{i k}$ is Kronecker delta, $F_{i j}$ is deformation gradient, $J=\operatorname{det} F_{i j}$ is Jacobian and $x_{i}$ is coordinate at present configuration. Eqs. (4) and (5) are unified to one equation as the following, 


$$
\rho_{0} \frac{\partial^{2} \dot{u}_{i}}{\partial t^{2}}-\rho_{0} \dot{f}_{i}=\frac{\partial}{\partial X_{j}}\left(A_{i j k l} \frac{\partial \dot{u}_{k}}{\partial x_{l}}\right)
$$

where tensor $A_{i j k l}$ is

$$
A_{i j k l}=J \frac{\partial X_{j}}{\partial x_{m}}\left(C_{i m k l}^{m a t}+\sigma_{l m} \delta_{i k}\right)
$$

It has been found that in Eq. (6), when the following condition is satisfied, arbitrariness appears in the mathematical solution of Eq. (6) [9].

$$
\operatorname{det}\left(A_{i j k} n_{j} n_{l}\right)=0
$$

Where ${ }^{n_{j}}$ is the vector having the angle of coordinate transformation. When we put the tensor $A_{i j k} n_{j} n_{l}$ as $a_{i k}$, the determinant of (8) is represented as the following,

$$
\operatorname{det} a_{i k}=0
$$

The above mathematical expressions are basically consistent for arbitrary deformation behaviour of composite materials including both longitudinal and transverse compressive failure modes, since the assumptions in the mathematical analysis are general cases including longitudinal and transverse compressive failure. In the following, the specific case for transverse compressive failure is considered. For considering transverse compressive failure, firstly the deformation behavior in transverse plane is assumed. Considering the two dimensional problem in transverse plane, and taking 2axis parallel to the vector ${ }^{n_{j}}, n_{j}$ becomes as follows,

$$
\left(\begin{array}{ll}
n_{2} & n_{3}
\end{array}\right)=\left(\begin{array}{ll}
1 & 0
\end{array}\right)
$$

Then Eq. (8) becomes as follows,

$$
\begin{aligned}
\operatorname{det}\left(A_{i j k} n_{j} n_{l}\right) & =\operatorname{det}\left(A_{i 2 k 2}\right) \\
& =\operatorname{det}\left(C_{i 2 k 2}^{\text {mat }}+\sigma_{22} \delta_{i k}\right)=0
\end{aligned}
$$

Solving for the determinant

$$
\left(C_{2222}^{\text {mat }}+\sigma_{22}\right)\left(C_{3232}^{\text {mat }}+\sigma_{22}\right)-C_{2232}^{\text {mat }} C_{3222}^{\text {mat }}=0
$$

Similar to the longitudinal compressive failure, the equation is expressed using the elastic-plastic tangent transverse shear modulus $G_{T T}^{e p}$, transverse tangent modulus $E_{T}^{e p}$ and in-plane Poisson's ratio $v_{12}$ and $v_{21}$,

$$
\left(\frac{1}{1-v_{12} v_{21}} E_{T}^{e p}+\sigma_{22}\right)\left(G_{T T}^{e p}+\sigma_{22}\right)-C_{2232}^{m a t} C_{3222}^{m a t}=0
$$

When $C_{2232}^{\text {mat }}$ and $C_{3222}^{\text {mat }}$ are close to zero, the equation becomes

$$
\left(\frac{1}{1-v_{12} v_{21}} E_{T}^{e p}+\sigma_{22}\right)\left(G_{T T}^{e p}+\sigma_{22}\right) \approx 0
$$

From this equation

$$
\frac{1}{1-v_{12} v_{21}} E_{T}^{e p}+\sigma_{22} \approx 0 \quad \text { or } G_{T T}^{e p}+\sigma_{22} \approx 0
$$

In the first case of Eq. (15),

$$
a_{22}=\frac{1}{1-v_{12} v_{21}} E_{T}^{e p}+\sigma_{22} \approx 0
$$

When $C_{2232}^{m a t}$ and $C_{3222}^{m a t}$ are close to zero, ${ }^{a_{23}}$ and $a_{32}$ are close to zero, and in this case the following equation is satisfied.

$$
\left(\begin{array}{cc}
0 & 0 \\
0 & a_{33}
\end{array}\right) \cdot\left(\begin{array}{l}
1 \\
0
\end{array}\right)=0 \cdot\left(\begin{array}{l}
1 \\
0
\end{array}\right)
$$

This equation indicates that the eigenvalue is zero and the eigenvector is $\left(\begin{array}{ll}1 & 0\end{array}\right)$

$$
\left(\begin{array}{ll}
v_{2} & v_{3}
\end{array}\right)=\left(\begin{array}{ll}
1 & 0
\end{array}\right)
$$

where $\left(\begin{array}{ll}v_{2} & v_{3}\end{array}\right)$ is the eigenvector. Between the eigenvector $\left(\begin{array}{ll}v_{2} & v_{3}\end{array}\right)$ and the vector $n_{j}$, there is the following relation.

$$
v_{i} / / n_{i}
$$

This case corresponds with the compaction band and the dilation band. From Eq. (16), the critical applied stress value is represented as follows,

$$
\sigma_{c r}=-\sigma_{22} \approx \frac{1}{1-v_{12} v_{21}} E_{T}^{e p}
$$

This represents the approximate expression of the material strength. In the second case of Eq. (15),

$$
a_{33}=G_{T T}^{e p}+\sigma_{22} \approx 0
$$

Similar to the previous case, when $C_{2232}^{\text {mat }}$ and $C_{3222}^{\text {mat }}$ are close to zero, ${ }^{a_{23}}$ and ${ }^{a_{32}}$ are close to zero, and then the following equation is satisfied.

$$
\left(\begin{array}{cc}
a_{22} & 0 \\
0 & 0
\end{array}\right) \cdot\left(\begin{array}{l}
0 \\
1
\end{array}\right)=0 \cdot\left(\begin{array}{l}
0 \\
1
\end{array}\right)
$$

This equation indicates that the eigenvalue is zero and the eigenvector is $\left(\begin{array}{ll}0 & 1\end{array}\right)$

$$
\left(\begin{array}{ll}
v_{2} & v_{3}
\end{array}\right)=\left(\begin{array}{ll}
0 & 1
\end{array}\right)
$$

In this case the relation between the eigenvector $\left(\begin{array}{ll}v_{2} & v_{3}\end{array}\right)$ and the vector ${ }_{j}$ becomes as follows

$$
v_{i} \perp n_{i} \quad \text { (24) }
$$

This case corresponds with the shear band. From Eq. (21), the critical applied stress value is represented as follows,

$$
\sigma_{c r}=-\sigma_{22} \approx G_{T T}^{e p}
$$

The critical stress value is also represented by the properties of fibers and matrix similar to the longitudinal compressive failure. From Eq. (2),

$$
\begin{aligned}
& G_{T T}^{e p} \approx G_{m}^{e p} \cdot \frac{\left(1-V_{f}\right)\left\{\left(G_{f T T}^{e}-G_{m}^{e p}\right) S_{T T}+G_{m}^{e p}\right\}+V_{f} G_{f T T}^{e}}{\left(1-V_{f}\right)\left(G_{f T T}^{e}-G_{m}^{e p}\right) S_{T T}+G_{m}^{e p}} \\
& \approx G_{m}^{e p} \cdot \frac{\left\{\left(1-V_{f}\right) S_{T T}+V_{f}\right\} G_{f T T}^{e}+\left(1-V_{f}\right)\left(1-S_{T T}\right) G_{m}^{e p}}{\left(1-V_{f}\right) S_{T T} G_{f T T}^{e}+\left\{1-\left(1-V_{f}\right) S_{T T}\right\} G_{m}^{e p}}
\end{aligned}
$$

where $G_{m}^{e p}$ is the elastic-plastic tangent shear modulus of matrix, $G_{f T T}^{e}$ is the elastic transverse shear modulus of fiber and $S_{T T}$ is the transverse shear component of Eshelby tensor. When the shear modulus of fiber is much higher than the shear modulus of matrix $G_{f T T}^{e} \gg G_{m}^{e p}$, 


$$
\begin{aligned}
& G_{T T}^{e p} \approx G_{m}^{e p} \cdot \frac{\left\{\left(1-V_{f}\right) S_{T T}+V_{f}\right\} G_{f T T}^{e}}{\left(1-V_{f}\right) S_{T T} G_{f T T}^{e}} \\
& \approx G_{m}^{e p} \cdot\left(1+\frac{V_{f}}{1-V_{f}} S_{T T}^{-1}\right)
\end{aligned}
$$

When fibers are the cylinder solids, the transverse shear component of the Eshelby tensor becomes

$$
S_{T T}=\frac{3-4 v_{m}}{4\left(1-v_{m}\right)}
$$

where ${ }^{v_{m}}$ is Poisson's ratio of matrix. Then Eq. (27) becomes

$$
G_{T T}^{e p} \approx G_{m}^{e p} \cdot\left(1+\frac{V_{f}}{1-V_{f}} \cdot \frac{4\left(1-v_{m}\right)}{3-4 v_{m}}\right)
$$

And Eq. (25) becomes

$$
\sigma_{c r} \approx\left(1+\frac{V_{f}}{1-V_{f}} \cdot \frac{4\left(1-v_{m}\right)}{3-4 v_{m}}\right) G_{m}^{e p}
$$

When fibers have the plate shape, the transverse shear component of the Eshelby tensor becomes $S_{T T}=1$. Then Eq. (27) becomes

$$
\begin{aligned}
& G_{T T}^{e p} \approx G_{m}^{e p} \cdot\left(1+\frac{V_{f}}{1-V_{f}}\right)=\frac{G_{m}^{e p}}{1-V_{f}} \\
& \sigma_{c r} \approx \frac{G_{m}^{e p}}{1-V_{f}}
\end{aligned}
$$

The equation is also solvable from the relationship between the elastic-plastic tangent shear modulus of matrix and the current yield state of matrix. From Eq. (3),

$$
d \tau_{m} \approx \frac{S_{T T} G_{f T T}^{e}+\left(1-S_{T T}\right) G_{m}^{e p}}{\left\{V_{f}+\left(1-V_{f}\right) S_{T T}\right\} G_{f T T}^{e}+\left(1-V_{f}\right)\left(1-S_{T T}\right) G_{m}^{e p}}
$$

where $d \tau_{m}$ is shear stress rate of matrix and $d \tau_{23, \text { comp }}$ is applied shear stress rate of composites. When the shear modulus of fiber is much higher than the shear modulus of

$$
\begin{aligned}
& \text { matrix } G_{f T T}^{e} \gg G_{m}^{e p} \text {, } \\
& d \tau_{m} \approx \frac{S_{T T} G_{f T T}^{e}}{\left\{V_{f}+\left(1-V_{f}\right) S_{T T}\right\} G_{f T T}^{e}} d \tau_{23, \text { comp }} \\
& \approx \frac{1}{1-V_{f}+V_{f} S_{T T}^{-1}} d \tau_{23, \text { comp }} \\
& d \tau_{23, \text { comp }} \approx\left(1-V_{f}+V_{f} S_{T T}{ }^{-1}\right) d \tau_{m}
\end{aligned}
$$

Then considering the integration until the time when the significant degradation of tangent modulus occurs,

$$
\tau_{23} \approx\left(1-V_{f}+V_{f} S_{T T}^{-1}\right) \tau_{m Y}
$$

where $\tau_{23}$ is applied shear stress to composites and $\tau_{m Y}$ is yield stress of matrix. Considering the equilibrium condition of applied stress in between the local coordinate system and the global coordinate system,

$$
\sigma_{y y} \sin 2 \theta / 2+\tau_{y z} \cos 2 \theta=\tau_{23}
$$

where $\sigma_{y y}$ and $\tau_{y z}$ are stress in transverse direction and transverse shear stress in the global coordinate system,

\begin{tabular}{|c|c|c|}
\hline Fiber volume fraction & $.00^{60}$ & $\%$ \\
\hline Matrix Poisson's ratio & $34^{0 .}$ & \\
\hline $\begin{array}{l}\text { Matrix yield stress } \\
\text { (shear) when nonlinearity } \\
\text { clearly appears }\end{array}$ & 70 & $\mathrm{~Pa}^{\mathrm{M}}$ \\
\hline Angle of shear band & 45 & $\mathrm{eg}^{\mathrm{d}}$ \\
\hline
\end{tabular}
respectively and $\theta$ is the angle between the local coordinate system and the global coordinate system. Solving for stress $\sigma_{y y}$

$$
\sigma_{c r}=\sigma_{y y} \approx \frac{\left(1-V_{f}+V_{f} S_{T T}^{-1}\right) \tau_{m Y}-\tau_{y z} \cos 2 \theta}{\sin 2 \theta / 2}
$$

Therefore the dependency of transverse compressive strength for the fiber volume fraction $V_{f}$ is related to $S_{T T}$. When fibers are plates, $S_{T T}^{-1}=1$ and

$$
\sigma_{c r} \approx \frac{2\left(\tau_{m Y}-\tau_{y z} \cos 2 \theta\right)}{\sin 2 \theta}
$$

And in the case of uniaxial compression

$$
\sigma_{x y} \approx \frac{2 \tau_{m Y}}{\sin 2 \theta}
$$

TABLE III. MATERIAL PROPERTY OF AS4/3501-6 [8].

On the other hand, when fibers are cylinder solids, $S_{T T}{ }^{-1}=4\left(1-v_{m}\right)\left(3-4 v_{m}\right)^{-1}$ and

$$
\begin{gathered}
\sigma_{c r} \approx\left\{\left(1-V_{f}+V_{f} \cdot \frac{4\left(1-v_{m}\right)}{3-4 v_{m}}\right) \tau_{m Y}-\tau_{y z} \cos 2 \theta\right\} \cdot \frac{2}{\sin 2 \theta} \\
\approx\left\{\left(1+\frac{V_{f}}{3-4 v_{m}}\right) \tau_{m Y}-\tau_{y z} \cos 2 \theta\right\} \cdot \frac{2}{\sin 2 \theta}
\end{gathered}
$$

In the case of uniaxial compression

$$
\sigma_{c r} \approx\left(1+\frac{V_{f}}{3-4 v_{m}}\right) \cdot \frac{2 \tau_{m Y}}{\sin 2 \theta}
$$

This equation indicates that the critical stress value is represented by fiber volume fraction, matrix Poisson's ratio, matrix yield stress and angle of shear band. Here the critical stress value is estimated for the actual material property using this equation. As the material, carbon fiber/epoxy resin AS4/3501-6 [8] is assumed. Table III shows the material property. Then the critical stress value is calculated as 191.2 MPa from Eq. (42). On the other hand, from Ref. [8], the transverse compressive strength of AS4/3501-6 is reported to be $200 \mathrm{MPa}$. Therefore the estimated value from Eq. (42) is close to the actual material strength value, which indicates that Eq. (42) is considered to be the approximate expression of material strength. 


\section{CONCLUSIONS}

The numerical simulation and theoretical modeling of transverse compressive failure are conducted. The critical stress value in transverse compressive failure is represented by fiber volume fraction, matrix Poisson's ratio, matrix yield stress and angle of shear band. The calculated critical stress value is close to the actual material strength value, and the expression for the critical stress value is considered to be the approximate expression of material strength.

\section{REFERENCES}

[1] M. J. Hinton and P. D. Soden, "Predicting Failure in Composite Laminates: The Background to The Exercise," Compos. Sci. Technol., vol. 58, pp. 1001-1010, 1998.

[2] C. R. Schultheisz and A. M. Waas, "Compressive Failure of Composites, Part I: Testing and Micromechanical Theories," Prog. Aerosp. Sci., vol. 32, pp. 1-42, 1996.

[3] A. M. Waas and C. R. Schultheisz, "Compressive Failure of Composites, Part II:, Experimental Studies” Prog. Aerosp. Sci., vol. 32, pp. 43-78, 1996.

[4] J. LLorca, C. González, J. M. Molina-Aldareguía, J. Segurado, R. Seltzer, F. Sket, M. Rodríguez, S. Sádaba, R. Muñoz, and L. P.
Canal, "Multiscale Modeling of Composite Materials: a Roadmap Towards Virtual Testing," Adv. Mater., vol. 23, pp. 5130-5147, 2011.

[5] D. Aragonés, "Fracture Micromechanisms in C/Epoxy Composites Under Transverse Compression" Master thesis, Universidad Politécnica de Madrid, 2007.

[6] A. Puck and H. Schürmann, "Failure Analysis of FRP Laminates By Means of Physically Based Phenomenological Models" Compos. Sci. Technol., vol. 62, pp. 1633-1662, 2002.

[7] E. Totry, C. González, J. LLorca, "Influence of the Loading Path On the Strength of Fiber-Reinforced Composites Subjected to Transverse Compression and Shear", Int. J. Solid. Struct., vol. 45, pp. 1663-1675, 2008.

[8] P. D. Soden, M. J. Hinton, and A. S. Kaddour, "Lamina Properties, Lay-up Configurations and Loading Conditions for a Range of Fibre-Reinforced Composite Laminates," Compos. Sci. Technol., vol. 58, pp. 1011-1022, 1998 .

[9] T. Nadabe, and N. Takeda, "Numerical Simulation and Theoretical Modeling of Longitudinal Compressive Failure in Fiber Reinforced Composite Materials," Proc. International Conference on Logistics Engineering, Management and Computer Science (LEMCS 2014), Atlantis-Press, May 2014, doi:10.2991/lemcs-14.2014.206.

[10] K. Tohgo, Y. Sugiyama, and K. Kawahara, "Ply-Cracking Damage and Nonlinear Deformation of CFRP Cross-Ply Laminate," JSME Int. J., Series A, vol. 45, pp. 545-552, 2002. 